\title{
Dimension of the space of intertwining operators from degenerate principal series representations
}

\author{
Taito Tauchi*
}

\begin{abstract}
Let $X$ be a homogeneous space of a real reductive Lie group $G$. It was proved by T. Kobayashi and T. Oshima that the regular representation $C^{\infty}(X)$ contains each irreducible representation of $G$ at most finitely many times if a minimal parabolic subgroup $P$ of $G$ has an open orbit in $X$, or equivalently, if the number of $P$-orbits on $X$ is finite. In contrast to the minimal parabolic case, for a general parabolic subgroup $Q$ of $G$, we find a new example that the regular representation $C^{\infty}(X)$ contains degenerate principal series representations induced from $Q$ with infinite multiplicity even when the number of $Q$-orbits on $X$ is finite.
\end{abstract}

Keywords: degenerate principal series, multiplicity, spherical variety, intertwining operators, real spherical.

MSC2010; primary 22E46; secondary 22E45, 53C30.

\section{Introduction}

Let $G$ be a real reductive algebraic Lie group, and $H$ an algebraic subgroup of $G$. T. Kobayashi and T. Oshima established the criterion of finite multiplicity for regular representations on $G / H$.

Fact 1.1 ([10, Theorem A]). The following two conditions on the pair $(G, H)$ are equivalent:

(i) $\operatorname{dim} \operatorname{Hom}_{G}\left(\pi, C^{\infty}(G / H, \tau)\right)<\infty$ for all $(\pi, \tau) \in \hat{G}_{\text {smooth }} \times \hat{H}_{\mathrm{f}}$.

(ii) $G / H$ is real spherical.

Here $\hat{G}_{\text {smooth }}$ denotes the set of equivalence classes of irreducible smooth admissible Fréchet representations of $G$ with moderate growth, and $\hat{H}_{\mathrm{f}}$ that of algebraic irreducible finite-dimensional representations of $H$. Given $\tau \in \hat{H}_{\mathrm{f}}$, we write $C^{\infty}(G / H, \tau)$ for the Fréchet space of smooth sections of the $G$-homogeneous vector bundle over $G / H$ associated to $\tau$. The terminology real sphericity was introduced by T. Kobayashi 8 in his search of a broader framework for global analysis on homogeneous spaces than the usual (e.g., reductive symmetric spaces).

${ }^{*}$ Graduate School of Mathematical Sciences, the University of Tokyo, Meguro-ku, Tokyo, 153-8914, Japan, E-mail address: taito@ms.u-tokyo.ac.jp 
Definition 1.2. A homogeneous space $G / H$ is real spherical if a minimal parabolic subgroup $P$ of $G$ has an open orbit in $G / H$.

The following equivalence is well known by the work of B. Kimelfeld [7] and the real rank one reduction of T. Matsuki [13]:

Fact 1.3 (3, Theorem 2.2]). $G / H$ is real spherical if and only if the number of $H$-orbits on $G / P$ is finite. In other words, the condition (ii) in Fact 1.1 is equivalent to the following condition (iii):

(iii) $\#(H \backslash G / P)<\infty$.

Therefore, for a minimal parabolic $P$, the three conditions (i), (ii), and (iii) are equivalent by Fact 1.1 and Fact 1.3 (see Figure 1.1 below). Then one might ask a question what will happen to the relationship among the three conditions, if we replace $P$ by a general parabolic subgroup $Q$ of $G$. For this, we need to make a precise definition of variants of (i), (ii), and (iii) for a parabolic subgroup $Q$ of $G$.

Definition 1.4 ([9, Definition 6.6]). We say $\pi \in \hat{G}_{\text {smooth }}$ belongs to $Q$-series if $\pi$ occurs as a subquotient of the degenerate principal series representation $C^{\infty}(G / Q, \tau)$ for some $\tau \in \hat{Q}_{\mathrm{f}}$.

$P$ : minimal parabolic

(i)

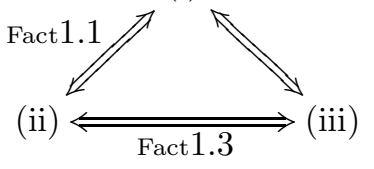

Figure 1.1
$Q$ : general parabolic

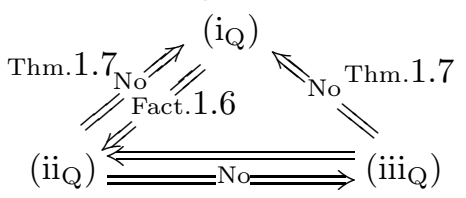

Figure 1.2

We set $\hat{G}_{\text {smooth }}^{Q}:=\left\{\pi \in \hat{G}_{\text {smooth }} \mid \pi\right.$ belongs to $Q$-series. $\}$. Obviously, $\hat{G}_{\text {smooth }}^{Q} \supset$ $\hat{G}_{\text {smooth }}^{Q^{\prime}}$ if $Q \subset Q^{\prime}$. Moreover, $\hat{G}_{\text {smooth }}^{Q}$ is equal to $\hat{G}_{\text {smooth }}$ if $Q=P$ (minimal parabolic) by Harish-Chandra's subquotient theorem [5] and to $\hat{G}_{\mathrm{f}}$ if $Q=G$.

Definition 1.5. For a parabolic subgroup $Q$ of $G$, we define the three conditions $\left(\mathrm{i}_{Q}\right),\left(\mathrm{ii}_{Q}\right)$, and $\left(\mathrm{iii}_{Q}\right)$, respectively, as follows:

$\left(\mathrm{i}_{Q}\right) \operatorname{dim} \operatorname{Hom}_{G}\left(\pi, C^{\infty}(G / H, \tau)\right)<\infty$ for all $(\pi, \tau) \in \hat{G}_{\text {smooth }}^{Q} \times \hat{H}_{\mathrm{f}}$.

(ii $Q) Q$ has an open orbit in $G / H$.

(iii $Q) \#(H \backslash G / Q)<\infty$.

The conditions $\left(\mathrm{i}_{Q}\right),\left(\mathrm{ii}_{Q}\right)$, and $\left(\mathrm{iii}_{Q}\right)$ reduce to (i), (ii), and (iii), respectively, if $Q=P$ (minimal parabolic), and we know from Fact 1.1 and Fact 1.3 (see also Figure 1.1) that the following equivalences hold:

$$
\left(\mathrm{i}_{Q}\right) \Longleftrightarrow\left(\mathrm{ii}_{Q}\right) \Longleftrightarrow\left(\mathrm{iii}_{Q}\right) \text { if } Q=P \text {. }
$$


Further, it is obvious from the Frobenius reciprocity that the condition $\left(\mathrm{i}_{Q}\right)$ automatically holds if $Q=G$; $\left(\mathrm{ii}_{Q}\right)$ and $\left(\mathrm{iii}_{Q}\right)$ obviously hold. Hence

$$
\left(\mathrm{i}_{Q}\right) \Longleftrightarrow\left(\mathrm{ii}_{Q}\right) \Longleftrightarrow\left(\mathrm{iii}_{Q}\right) \text { if } Q=G \text {. }
$$

In the general setting, clearly, $\left(\mathrm{iii}_{Q}\right)$ implies $\left(\mathrm{ii}_{Q}\right)$, however the converse may fail if $Q$ is not a minimal parabolic subgroup of $G$. On the other hand, the implication $\left(\mathrm{i}_{Q}\right) \Rightarrow\left(\mathrm{ii}_{Q}\right)$ is true. In fact, the following stronger theorem holds:

Fact 1.6 ([9, Corollary 6.8]). If there exists $\tau \in \hat{H}_{\mathrm{f}}$ such that for all $\pi \in \hat{G}_{\mathrm{smooth}}^{Q}$ $\operatorname{dim} \operatorname{Hom}_{G}\left(\pi, C^{\infty}(G / H, \tau)\right)<\infty$, then $\left(\mathrm{ii}_{Q}\right)$ holds.

An open problem is whether the converse statement holds or not.

Question. Does the finite-multiplicity condition $\left(\mathrm{i}_{Q}\right)$ in representation theory follows from the geometric condition $\left(\mathrm{ii}_{Q}\right)\left(\right.$ or $\left.\left(\mathrm{iii}_{Q}\right)\right)$ ?

We give a negative answer to this question in this paper. Explicitly, we prove the theorem below:

Theorem 1.7. Let $Q$ be a maximal parabolic subgroup of $G=S L(2 n, \mathbb{R})$ such that $G / Q$ is isomorphic to the real projective space $\mathbb{R}^{2 n-1}$. Then if $n \geq 2$, there exists an algebraic subgroup $H$ of $G$ satisfying the following two conditions:

1) $\#(H \backslash G / Q)<\infty$,

2) $\operatorname{dim} \operatorname{Hom}_{G}\left(C^{\infty}(G / Q, \chi), C^{\infty}(G / H)\right)=\infty$ for some one-dimensional representation $\chi$ of $Q$.

Furthermore, if $n \geq 3, H$ satisfies the following condition:

$\left.2^{\prime}\right) \operatorname{dim} \operatorname{Hom}_{G}\left(C^{\infty}(G / Q, \chi), C^{\infty}(G / H)\right)=\infty$ for any one-dimensional representation $\chi$ of $Q$.

We summarize the relationship among the conditions $\left(\mathrm{i}_{Q}\right),\left(\mathrm{ii}_{Q}\right)$, and $\left(\mathrm{iii}_{Q}\right)$ as follows: $\left(\mathrm{i}_{Q}\right) \Rightarrow\left(\mathrm{ii}_{Q}\right)$ is true by Fact 1.6. Theorem 1.7 implies that neither $\left(\mathrm{iii}_{Q}\right) \Rightarrow\left(\mathrm{i}_{Q}\right)$ nor $\left(\mathrm{ii}_{Q}\right) \Rightarrow\left(\mathrm{i}_{Q}\right)$ holds, see Figure 1.2.

Remark 1.8. The recent paper [2, Theorem D] claimed the following: Suppose that a real algebraic group $H$ acts on a real algebraic smooth variety $M$ with $\#(H \backslash M)<\infty$ and that $E$ is an algebraic $H$-homogeneous vector bundle on $M$. Then, for any $n \in \mathbb{N}$,

$$
\sup _{\substack{\tau \in \hat{H}_{\mathrm{f}} \\ \operatorname{dim} \tau=n}} \operatorname{dim} \operatorname{Hom}_{H}\left(\tau, \mathcal{S}^{*}(M, E)\right)<\infty .
$$

We note that $\mathcal{S}^{*}(M, E)$ can be identified with the space $\mathcal{D}^{\prime}(M)$ of distributions in the case that $M$ is compact and $E$ is the trivial bundle $M \times \mathbb{C}[1$, Chapter 1.5]. Therefore (1.1) would imply

$$
\operatorname{dim} \operatorname{Hom}_{H}\left(\mathbf{1}, \mathcal{D}^{\prime}(M)\right)=\operatorname{dim} \mathcal{D}^{\prime}(M)^{H}<\infty,
$$


when $\#(H \backslash M)<\infty$ and $M$ is compact. Here $\mathbf{1}$ denotes the trivial onedimensional representation of $H$.

However, one sees from Fact 2.2 that (1.2) contradicts to Theorem 1.7 when applied to $M=\mathbb{R} \mathbb{P}^{2 n-1}$. Thus Theorem 1.7 is a counterexample to [2, Theorem D]. Indeed, it seems to the author that a gap in the proof of [2, Theorem D] comes from a false statement $\#(H \backslash G / Q)<\infty \Rightarrow \#\left(H_{\mathbb{C}} \backslash G_{\mathbb{C}} / Q_{\mathbb{C}}\right)<\infty$, see Remark 4.9 below.

The outline of this article as follows: In Section 2, we recall some general facts concerning distribution kernels, which were proved by T. Kobayashi and B. Speh [1]. In Section 3, we fix some basic notation for distributions on the complex Euclidean space. In Section 4 , we construct the subgroup $H$ of $G$ and give a proof of Theorem 1.7 .

\section{Reduction to distribution kernels}

In this section, we reformulate the condition 2) of Theorem 1.7 by means of distribution kernels using Fact 2.2 below.

Definition 2.1. Let $G$ be a real Lie group and $H$ a closed subgroup of $G$. For $\tau \in \hat{H}_{\mathrm{f}}$, we define the finite-dimensional representation of $H$ by $\tau_{2 \rho}^{\vee}:=\tau^{\vee} \otimes \mathbb{C}_{2 \rho}$ where $\tau^{\vee}$ is the contragredient representation of $\tau$ and $\mathbb{C}_{2 \rho}$ denotes the onedimensional representation of $H$ given by $h \mapsto|\operatorname{det}(\operatorname{Ad}(h): \mathfrak{g} / \mathfrak{h} \rightarrow \mathfrak{g} / \mathfrak{h})|^{-1}$.

Fact 2.2 ([11, Proposition 3.2]). Let $G$ be a real Lie group. Suppose that $G^{\prime}$ and $H$ are closed subgroups of $G$ and that $H^{\prime}$ is a closed subgroup of $G^{\prime}$. Let $\tau$ and $\tau^{\prime}$ be finite-dimensional representations of $H$ and $H^{\prime}$, respectively.

(1) There is a natural injective map:

$$
\operatorname{Hom}_{G^{\prime}}\left(C^{\infty}(G / H, \tau), C^{\infty}\left(G^{\prime} / H^{\prime}, \tau^{\prime}\right)\right) \hookrightarrow\left(\mathcal{D}^{\prime}\left(G / H, \tau_{2 \rho}^{\vee}\right) \otimes \tau^{\prime}\right)^{H^{\prime}} .
$$

Here $\left(\mathcal{D}^{\prime}\left(G / H, \tau_{2 \rho}^{\vee}\right) \otimes \tau^{\prime}\right)^{H^{\prime}}$ denotes the space of $H^{\prime}$-fixed vectors under the diagonal action.

(2) If $H$ is cocompact in $G$ (e.g., a parabolic subgroup of $G$ or a uniform lattice), then (2.1) is a bijection.

We apply this fact to the setting of Theorem 1.7 Recall that $G=S L(2 n, \mathbb{R})$ and $Q$ is a maximal parabolic subgroup of $G$ such that $G / Q \simeq \mathbb{R} \mathbb{P}^{2 n-1}$. For $\lambda \in \mathbb{C}$, we define a one-dimensional representation $\chi_{\lambda}: Q \rightarrow G L(1, \mathbb{C})$ by $g \mapsto|\operatorname{det}(\operatorname{Ad}(g): \mathfrak{g} / \mathfrak{q} \rightarrow \mathfrak{g} / \mathfrak{q})|^{\frac{-\lambda}{2 n}}$. We denote by $\mathcal{D}^{\prime}\left(\mathbb{R}^{2 n} \backslash\{0\}\right)_{\text {even, },-2 n}$ the space of even homogeneous distributions of degree $\lambda-2 n$ on $\mathbb{R}^{2 n} \backslash\{0\}$.

Corollary 2.3. For any closed subgroup $H$ of $G$, we have

$$
\operatorname{Hom}_{G}\left(C^{\infty}\left(G / Q, \chi_{\lambda}\right), C^{\infty}(G / H)\right) \simeq \mathcal{D}^{\prime}\left(\mathbb{R}^{2 n} \backslash\{0\}\right)_{\text {even }, \lambda-2 n}^{H} .
$$

Proof. This follows from Fact 2.2 because $\mathbb{C}_{2 \rho}=\chi_{2 n}$ as representations of $Q$

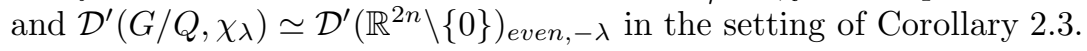




\section{Notation for distributions on the complex Eu- clidean space}

In Section 4, we shall consider a linear group action on $\mathbb{C}^{n}$ regarded as a real vector space. In order to avoid possible confusion, we prepare some notation for distributions on the complex Euclidean space $\mathbb{C}^{n}$ regarded as a real vector space. Identifying $\mathbb{C}^{n}$ with $\mathbb{R}^{2 n}$ by $z=\left(z_{1}, \ldots, z_{n}\right)=\left(x_{1}+i y_{1}, \ldots, x_{n}+i y_{n}\right)$, we write $\mathcal{D}\left(\mathbb{C}^{n}\right)$ and $\mathcal{D}^{\prime}\left(\mathbb{C}^{n}\right)$ for the spaces of $C^{\infty}$ functions with compact support and distributions on $\mathbb{C}^{n} \simeq \mathbb{R}^{2 n}$, respectively. We define a distribution $\delta\left(z_{n}, \bar{z}_{n}\right) \in$ $\mathcal{D}^{\prime}\left(\mathbb{C}^{n}\right) \simeq \mathcal{D}^{\prime}\left(\mathbb{R}^{2 n}\right)$ by

$$
\begin{aligned}
\delta\left(z_{n}, \bar{z}_{n}\right)(\phi) & :=\frac{1}{(-2 i)^{n}} \int_{\mathbb{C}^{n-1}} \phi\left(z_{1}, \ldots, z_{n-1}, 0\right) d z_{1} d \bar{z}_{1} \ldots d z_{n-1} d \bar{z}_{n-1} \\
& =\frac{1}{-2 i} \int_{\mathbb{R}^{2 n-2}} \phi\left(x^{\prime}+i y^{\prime}, 0\right) d x_{1} d y_{1} \ldots d x_{n-1} d y_{n-1}
\end{aligned}
$$

for every test function $\phi \in \mathcal{D}\left(\mathbb{C}^{n}\right) \simeq \mathcal{D}\left(\mathbb{R}^{2 n}\right)$ where $x^{\prime}+i y^{\prime}:=\left(x_{1}+i y_{1}, \ldots, x_{n-1}+\right.$ $\left.i y_{n-1}\right)$. We write $\delta(\cdot)$ for the usual Dirac delta function on $\mathbb{R}$ and regard it as a distribution on $\mathbb{R}^{2 n}$ by the pull-back via the projection $\mathbb{R}^{2 n} \rightarrow \mathbb{R}$. Then we have

$$
\delta\left(z_{n}, \bar{z}_{n}\right)=(-2 i)^{-1} \delta\left(x_{n}\right) \delta\left(y_{n}\right)
$$

as distributions on $\mathbb{C}^{n} \simeq \mathbb{R}^{2 n}$. Since the multiplication by $x_{n}$ or $y_{n}$ kills (3.1), so does it by $z_{n}$ or $\bar{z}_{n}=x_{n}-i y_{n}$, that is,

$$
z_{n} \delta\left(z_{n}, \bar{z}_{n}\right)=\bar{z}_{n} \delta\left(z_{n}, \bar{z}_{n}\right)=0 .
$$

We define differential operators on $\mathbb{C}^{n} \simeq \mathbb{R}^{2 n}$ by

$$
\frac{\partial}{\partial z_{j}}:=\frac{1}{2}\left(\frac{\partial}{\partial x_{j}}-i \frac{\partial}{\partial y_{j}}\right), \quad \frac{\partial}{\partial \bar{z}_{j}}:=\frac{1}{2}\left(\frac{\partial}{\partial x_{j}}+i \frac{\partial}{\partial y_{j}}\right) \quad(1 \leq j \leq n) .
$$

Multiplication of $\frac{\partial^{l}}{\partial z_{n}^{l}} \delta\left(z_{n}, \bar{z}_{n}\right)$ by distributions of $z_{1}, \bar{z}_{1}, \ldots, z_{n-1}, \bar{z}_{n-1}$ makes sense. We note that a finite family $\left\{T_{l}\right\}_{l=1}^{m}$ of distributions on $\mathbb{C}^{n-1} \backslash\{0\}$ vanish if the following equality as distributions on $\mathbb{C}^{n} \backslash\{0\} \simeq \mathbb{R}^{2 n} \backslash\{0\}$ holds:

$$
\sum_{l=1}^{m} T_{l}\left(z_{1}, \ldots, z_{n-1}\right) \frac{\partial^{l}}{\partial z_{n}^{l}} \delta\left(z_{n}, \bar{z}_{n}\right)=0 .
$$

Suppose a group $G$ acts linearly on $\mathbb{C}^{n}$ regarded as a real vector space. In turn, $G$ acts on the spaces of $C^{\infty}$ functions $f$, distributions $T$, and differential operators $D$ on $\mathbb{C}^{n} \simeq \mathbb{R}^{2 n}$. We shall denote these actions by

$$
\begin{aligned}
(g \cdot f)(z) & :=f\left(g^{-1} \cdot z\right), \\
(g \cdot T)(\phi) & :=T\left(g^{-1} \cdot \phi\right), \\
(g \cdot D)(f) & :=g \cdot\left(D\left(g^{-1} \cdot f\right)\right),
\end{aligned}
$$

where $g \in G, z \in \mathbb{C}^{n}$, and $\phi \in \mathcal{D}\left(\mathbb{C}^{n}\right) \simeq \mathcal{D}\left(\mathbb{R}^{2 n}\right)$. 


\section{Proof of Theorem 1.7}

In this section, we take $G$ to be $S L(2 n, \mathbb{R})$, and construct an algebraic subgroup $H$ satisfying the two conditions 1) and 2) in Theorem 1.7 We begin with a 4-dimensional $\mathbb{R}$-algebra $R_{\varepsilon}$ defined by

$$
\begin{aligned}
R_{\varepsilon} & :=\mathbb{C} \oplus \mathbb{C} \varepsilon & & \text { as a vector } \\
(a+b \varepsilon)(c+d \varepsilon) & :=(a c+b \bar{d})+(b \bar{c}+a d) \varepsilon & & \text { as a ring, }
\end{aligned}
$$

with $\varepsilon$ being just a symbol, and $a, b, c, d \in \mathbb{C}$. Regarding $\mathbb{C}$ as an $\mathbb{R}$-vector space, we let $R_{\varepsilon}$ act $\mathbb{R}$-linearly on $\mathbb{C}$ by

$$
(a+b \varepsilon) \cdot z:=a z+b \bar{z} \quad\left(a+b \varepsilon \in R_{\varepsilon}, z \in \mathbb{C}\right) .
$$

Remark 4.1. We write $i$ for the imaginary unit of $\mathbb{C}$, then by (4.1) we have

$$
\varepsilon^{2}=1, \quad i^{2}=-1, \quad i \varepsilon=-\varepsilon i .
$$

Therefore $R_{\varepsilon}$ is isomorphic to the real Clifford algebra $C(1,1)$ as an $\mathbb{R}$-algebra. Hence we have $R_{\varepsilon} \simeq C(1,1) \simeq M_{2}(\mathbb{R})$ (for example, [12, Proposition 4.4.1]).

Let $M_{n}\left(R_{\varepsilon}\right)$ be the $\mathbb{R}$-algebra of all $n \times n$ matrices over $R_{\varepsilon}$. The left multiplication defines a (real) representation of $M_{n}\left(R_{\varepsilon}\right)$ on $\mathbb{C}^{n}$ regarded as a vector space over $\mathbb{R}$. This representation induces an injective $\mathbb{R}$-algebra homomorphism

$$
\iota: M_{n}\left(R_{\varepsilon}\right) \hookrightarrow M_{2 n}(\mathbb{R}),
$$

which is also surjective because the real dimensions of $M_{n}\left(R_{\varepsilon}\right)$ and $M_{2 n}(\mathbb{R})$ are the same. We define a subgroup $H$ of $M_{n}\left(R_{\varepsilon}\right)$ by

$$
H:=\left\{h^{\theta}(\boldsymbol{a}):=\left(\begin{array}{ccccc}
e^{i \theta} & a_{1} \varepsilon & a_{2} \varepsilon^{2} & \cdots & a_{n-1} \varepsilon^{n-1} \\
& e^{i \theta} & a_{1} \varepsilon & \ddots & \vdots \\
& & e^{i \theta} & \ddots & a_{2} \varepsilon^{2} \\
& & & \ddots & a_{1} \varepsilon \\
& & & & e^{i \theta}
\end{array}\right) \mid \begin{array}{c}
\boldsymbol{a} \in \mathbb{C}^{n-1} \\
\theta \in \mathbb{R}
\end{array}\right\},
$$

where $\boldsymbol{a}=\left(a_{1}, \ldots, a_{n-1}\right) \in \mathbb{C}^{n-1}$. Then $\iota(H)$ is a subgroup of $G L(2 n, \mathbb{R})$.

Lemma 4.2. $\operatorname{det}(\iota(H))=\{1\}$.

Proof. For any $\boldsymbol{a} \in \mathbb{C}^{n-1}$, it is clear that $\operatorname{det}\left(\iota\left(h^{0}(\boldsymbol{a})\right)\right)=1$ since $\iota\left(h^{0}(a)\right) \in$ $G L(2 n, \mathbb{R})$ is a unipotent matrix. Moreover dividing $\iota\left(h^{\theta}(0, \ldots, 0)\right) \in G L(2 n, \mathbb{R})$ into $2 \times 2$ block matrices, we have $\operatorname{det}\left(\iota\left(h^{\theta}(0, \ldots, 0)\right)\right)=1$ for any $\theta \in \mathbb{R}$ because $e^{i \theta}$ acts on $\mathbb{C} \simeq \mathbb{R}^{2}$ as rotation. Since the group $H$ is generated by elements of the form $h^{0}(\boldsymbol{a})$ and $h^{\theta}(0, \ldots, 0)$, the lemma is proved. 

via $\iota$.

By Lemma 4.2, we may identify $H$ in $M_{n}\left(R_{\varepsilon}\right)$ with $\iota(H)$ in $G=S L(2 n, \mathbb{R})$

The following proposition shows that the subgroup $H$ of $G$ satisfies the condition 1) in Theorem 1.7.

Proposition 4.3. For every $j \in\{1,2, \ldots, n\}$, there exists exactly one $H$-orbit on $G / Q$ of real dimension $2 j-1$. These orbits exhaust all $H$-orbits on $G / Q$. In particular, \# $(H \backslash G / Q)=n<\infty$.

Proof. Let $\mathbb{R}^{\times}:=G L(1, \mathbb{R})$ act on $\mathbb{C}^{n}$ by scalar multiplication and put $X:=$ $\left(\mathbb{C}^{n} \backslash\{0\}\right) / \mathbb{R}^{\times}$. Identifying $\mathbb{C}^{n}$ with $\mathbb{R}^{2 n}$, we have $X \simeq \mathbb{R} \mathbb{P}^{2 n-1} \simeq G / Q$ and these isomorphisms induce a bijection:

$$
H \backslash X \simeq H \backslash G / Q
$$

For $j \in\{1,2, \ldots, n\}$, we define a real $(2 j-1)$-dimensional submanifold of $X$ by

$$
Y_{2 j-1}:=\left\{\left(z_{1}, \ldots, z_{j}, 0, \ldots, 0\right) \in \mathbb{C}^{n} \mid z_{j} \neq 0\right\} / \mathbb{R}^{\times} \subset X .
$$

Then the group $H$ leaves $Y_{2 j-1}$ invariant, and in fact it acts transitively. Thus we have an orbit decomposition

$$
H \backslash X=\bigcup_{j=1}^{n} Y_{2 j-1}
$$

Therefore $\#(H \backslash G / Q)=\#(H \backslash X)=n<\infty$.

Let us prove that the subgroup $H$ of $G$ satisfies the condition 2') of Theorem 1.7 in the case of $n \geq 3$. We define two real analytic vector fields $D$ and $\bar{D}$ on $\mathbb{C}^{n} \simeq \mathbb{R}^{2 n}$ for $n \geq 3$ by

$$
D:=\bar{z}_{n-2} \frac{\partial}{\partial \bar{z}_{n-1}}+z_{n-1} \frac{\partial}{\partial z_{n}}, \quad \bar{D}:=z_{n-2} \frac{\partial}{\partial z_{n-1}}+\bar{z}_{n-1} \frac{\partial}{\partial \bar{z}_{n}} .
$$

For $l \in \mathbb{N}$, we define nonzero two distributions $T_{\lambda}^{l}, \bar{T}_{\lambda}^{l} \in \mathcal{D}^{\prime}\left(\mathbb{C}^{n} \backslash\{0\}\right)$ with holomorphic parameter $\lambda \in \mathbb{C}$ by

$$
\begin{aligned}
T_{\lambda}^{l}(z) & :=\frac{1}{\Gamma\left(2-\frac{\lambda}{2}\right)} D^{l}\left(\left|z_{n-1}\right|^{2-\lambda} \delta\left(z_{n}, \bar{z}_{n}\right)\right), \\
\bar{T}_{\lambda}^{l}(z) & :=\frac{1}{\Gamma\left(2-\frac{\lambda}{2}\right)} \bar{D}^{l}\left(\left|z_{n-1}\right|^{2-\lambda} \delta\left(z_{n}, \bar{z}_{n}\right)\right),
\end{aligned}
$$

where $\Gamma(\cdot)$ denotes the gamma function. We note that $\left|z_{n-1}\right|^{2-\lambda}=\left(x_{n-1}^{2}+\right.$ $\left.y_{n-1}^{2}\right)^{1-\frac{\lambda}{2}}$ has a simple pole at $\lambda \in 2 \mathbb{N}+4$ as a distribution and $\Gamma\left(2-\frac{\lambda}{2}\right)$ has a simple pole at $\lambda \in 2 \mathbb{N}+4$. Therefore $T_{\lambda}^{l}$ and $\bar{T}_{\lambda}^{l}$ define distributions with holomorphic parameter $\lambda \in \mathbb{C}$ (for example, see [4, Appendix B1.4]). Moreover 
$T_{\lambda}^{l}$ and $\bar{T}_{\lambda}^{l}$ are homogeneous distributions of degree $-\lambda$ because $\left|z_{n-1}\right|^{2-\lambda}$ and $\delta\left(z_{n}, \bar{z}_{n}\right)$ are homogeneous of degree $2-\lambda$ and -2 , respectively, and the operators $D$ and $\bar{D}$ preserve the degrees. Clearly, $T_{\lambda}^{l}$ and $\bar{T}_{\lambda}^{l}$ are even distributions, therefore $T_{\lambda}^{l}, \bar{T}_{\lambda}^{l} \in \mathcal{D}^{\prime}\left(\mathbb{C}^{n} \backslash\{0\}\right)_{\text {even },-\lambda} \simeq \mathcal{D}^{\prime}\left(G / Q, \chi_{\lambda}\right)$.

Proposition 4.4. Suppose $n \geq 3$. Then for any $\lambda \in \mathbb{C}$ and any $l \in \mathbb{N}$, the distributions $T_{\lambda}^{l}$ and $\bar{T}_{\lambda}^{l}$ are $H$-invariant, that is, $T_{\lambda}^{l}, \bar{T}_{\lambda}^{l} \in \mathcal{D}^{\prime}\left(\mathbb{C}^{n} \backslash\{0\}\right)_{\text {even },-\lambda}^{H}$.

Proof. We prove only the claim for $T_{\lambda}^{l}$ as that for $\bar{T}_{\lambda}^{l}$ can be shown similarly. We define elements of $H$ by the equality

$$
h(\theta):=h^{\theta}(0, \ldots, 0), \quad h_{j}(a):=h^{0}(0, \ldots, 0, \stackrel{j}{\vee}, 0, \ldots, 0),
$$

where $\theta \in \mathbb{R}, a \in \mathbb{C}$ and, $j \in\{1,2, \ldots, n-1\}$ (see (4.4) for notation). Then it is sufficient to prove that $h(\theta) \cdot T_{\lambda}^{l}=T_{\lambda}^{l}$ for any $\theta \in \mathbb{R}$ and $h_{j}(a) \cdot T_{\lambda}^{l}=T_{\lambda}^{l}$ for any $a \in \mathbb{C}$ and $j \in\{1,2, \ldots, n-1\}$ because the group $H$ is generated by elements of the form $h(\theta)$ and $h_{j}(a)$. The first claim follows easily from $h(\theta) \cdot z=e^{i \theta} z$ for $z \in \mathbb{C}^{n}$. For the case of $j=1$ of the second claim, we need the following:

Lemma 4.5. Let $D$ be the vector field defined in (4.7). Then, we have

$$
h_{1}(a) \cdot D=D+a\left(\bar{z}_{n-2}-\bar{a} z_{n-1}+|a|^{2} \bar{z}_{n}\right) \frac{\partial}{\partial z_{n-2}}-a \bar{z}_{n} \frac{\partial}{\partial z_{n}} \quad(a \in \mathbb{C}) .
$$

This is an easy calculation, hence we omit the proof.

By Lemma 4.5, the following equality as distributions on $\mathbb{C}^{n} \backslash\{0\} \simeq \mathbb{R}^{2 n} \backslash\{0\}$ holds:

$$
\begin{aligned}
\left(h_{1}(a) \cdot T_{\lambda}^{l}\right)(z) & =\frac{1}{\Gamma\left(2-\frac{\lambda}{2}\right)}\left(h_{1}(a) \cdot D\right)^{l}\left(\left|z_{n-1}-a \bar{z}_{n}\right|^{2-\lambda} \delta\left(z_{n}, \bar{z}_{n}\right)\right) \\
& =\frac{1}{\Gamma\left(2-\frac{\lambda}{2}\right)}\left(D-a \bar{z}_{n} \frac{\partial}{\partial z_{n}}\right)^{l}\left(\left|z_{n-1}\right|^{2-\lambda} \delta\left(z_{n}, \bar{z}_{n}\right)\right) \\
& =\frac{1}{\Gamma\left(2-\frac{\lambda}{2}\right)} D^{l}\left(\left|z_{n-1}\right|^{2-\lambda} \delta\left(z_{n}, \bar{z}_{n}\right)\right) \\
& =T_{\lambda}^{l}(z) .
\end{aligned}
$$

We have used (3.2) and $\frac{\partial}{\partial z_{n-2}}\left(\left|z_{n-1}\right|^{2-\lambda} \delta\left(z_{n}, \bar{z}_{n}\right)\right)=0$ in the second equality. For $j \in\{2,3, \ldots, n-1\}, h_{j}(a) \cdot T_{\lambda}^{l}=T_{\lambda}^{l}$ can be shown similarly in the case $j=1$. Therefore $T_{\lambda}^{l}$ is $H$-invariant. Thus the proof of proposition completes.

Proposition 4.6. If $n \geq 3$, for any $\lambda \in \mathbb{C}$ we have

$$
\operatorname{dim} \mathcal{D}^{\prime}\left(\mathbb{C}^{n} \backslash\{0\}\right)_{\text {even },-\lambda}^{H}=\infty .
$$


Proof. We know from Proposition 4.4 that $T_{\lambda}^{l} \in \mathcal{D}^{\prime}\left(\mathbb{C}^{n} \backslash\{0\}\right)_{\text {even },-\lambda}^{H}$ for all $l \in \mathbb{N}$. Therefore it is sufficient to prove that $\left\{T_{\lambda}^{l}\right\}_{l \in \mathbb{N}}$ is linearly independent. But this is a consequence of (3.3) and the following equality as distributions on $\mathbb{C}^{n} \backslash\{0\} \simeq \mathbb{R}^{2 n} \backslash\{0\}:$

$$
\begin{aligned}
T_{\lambda}^{l}(z) & =\frac{1}{\Gamma\left(2-\frac{\lambda}{2}\right)}\left(\bar{z}_{n-2} \frac{\partial}{\partial \bar{z}_{n-1}}+z_{n-1} \frac{\partial}{\partial z_{n}}\right)^{l}\left(\left|z_{n-1}\right|^{2-\lambda} \delta\left(z_{n}, \bar{z}_{n}\right)\right) \\
& =\frac{1}{\Gamma\left(2-\frac{\lambda}{2}\right)} \sum_{k=0}^{l}\left(\begin{array}{l}
l \\
k
\end{array}\right)\left(\bar{z}_{n-2} \frac{\partial}{\partial \bar{z}_{n-1}}\right)^{k}\left(z_{n-1} \frac{\partial}{\partial z_{n}}\right)^{l-k}\left(\left|z_{n-1}\right|^{2-\lambda} \delta\left(z_{n}, \bar{z}_{n}\right)\right) \\
& =\frac{1}{\Gamma\left(2-\frac{\lambda}{2}\right)} \sum_{k=0}^{l}\left(\begin{array}{l}
l \\
k
\end{array}\right)\left(\bar{z}_{n-2}^{k} z_{n-1}^{l-k} \frac{\partial^{k}\left|z_{n-1}\right|^{2-\lambda}}{\partial \bar{z}_{n-1}^{k}}\right) \frac{\partial^{l-k}}{\partial z_{n}^{l-k}} \delta\left(z_{n}, \bar{z}_{n}\right) .
\end{aligned}
$$

We have used the binomial expansion in the second equality.

Proof of Theorem 1.7 in the case $n \geq 3$. We take $H$ to be the subgroup (4.4) via the inclusion $\iota$ (4.3). Then $H$ satisfies 1) by Proposition 4.3. Moreover $H$ satisfies 2') by Corollary 2.3 and Proposition 4.6 because $\mathcal{D}^{\prime}\left(\mathbb{R}^{2 n} \backslash\{0\}\right)_{\text {even, },-\lambda}^{H} \simeq$ $\mathcal{D}^{\prime}\left(\mathbb{C}^{n} \backslash\{0\}\right)_{\text {even },-\lambda}^{H}$. We note that any one-dimensional representation $\chi$ of $Q$ is of the form $\chi_{\lambda}$ for some $\lambda \in \mathbb{C}$.

Next we discuss in the case $n=2$. For $\lambda=2$ in (4.8) and (4.9), the binomial expansion shows

$$
\begin{aligned}
T_{2}^{l}(z) & =\left(\bar{z}_{n-2} \frac{\partial}{\partial \bar{z}_{n-1}}+z_{n-1} \frac{\partial}{\partial z_{n}}\right)^{l} \delta\left(z_{n}, \bar{z}_{n}\right) \\
& =\left(z_{n-1} \frac{\partial}{\partial z_{n}}\right)^{l} \delta\left(z_{n}, \bar{z}_{n}\right), \\
\bar{T}_{2}^{l}(z) & =\left(\bar{z}_{n-1} \frac{\partial}{\partial \bar{z}_{n}}\right)^{l} \delta\left(z_{n}, \bar{z}_{n}\right) .
\end{aligned}
$$

In the second equality, we have used $\frac{\partial}{\partial \bar{z}_{n-1}} \delta\left(z_{n}, \bar{z}_{n}\right)=0$ because $\delta\left(z_{n}, \bar{z}_{n}\right)$ does not depend on the variable $\bar{z}_{n-1}$. Then we define $T_{2}^{l}$ and $\bar{T}_{2}^{l}$ in the case of $(n, \lambda)=(2,2)$ by (4.11) and (4.12), respectively, in which the variables $z_{n-2}, \bar{z}_{n-2}$ do not appear. By using these distributions, we prove the case $n=2$ of Theorem 1.7 .

Proof of Theorem 1.7 in the case of $n=2$. We take $H$ to be the subgroup (4.4) via the inclusion $\iota(4.3)$ as in the case of $n \geq 3$, then $H$ satisfies 1 ) by Proposition 4.3. Set $D^{\prime}:=z_{1} \frac{\partial}{\partial z_{2}}$. By (4.11) we have

$$
T_{2}^{l}(z)=\left(D^{\prime}\right)^{l} \delta\left(z_{2}, \bar{z}_{2}\right) .
$$


We note that the group $H$ is generated by elements of the form $h(\theta)$ and $h_{1}(a)$ in the case of $n=2$. Just like before, $h(\theta) \cdot T_{\lambda}^{l}=T_{\lambda}^{l}$ follows from $h(\theta) \cdot z=e^{i \theta} z$ for $z \in \mathbb{C}^{2}$. Moreover, direct computation shows

$$
h_{1}(a) \cdot D^{\prime}=D^{\prime}+\bar{a}\left(z_{1}-a \bar{z}_{2}\right) \frac{\partial}{\partial \bar{z}_{1}}-a \bar{z}_{2} \frac{\partial}{\partial z_{2}} \quad(a \in \mathbb{C}) .
$$

Hence in the same way as in $n \geq 3$, the following equality of distributions on $\mathbb{C}^{2} \backslash\{0\} \simeq \mathbb{R}^{4} \backslash\{0\}$ holds:

$$
\begin{aligned}
h_{1}(a) \cdot T_{2}^{l}(z) & =\left(D^{\prime}+\bar{a}\left(z_{1}-a \bar{z}_{2}\right) \frac{\partial}{\partial \bar{z}_{1}}-a \bar{z}_{2} \frac{\partial}{\partial z_{2}}\right)^{l} \delta\left(z_{2}, \bar{z}_{2}\right) \\
& =\left(D^{\prime}\right)^{l} \delta\left(z_{n}, \bar{z}_{n}\right) \\
& =T_{2}^{l}(z) .
\end{aligned}
$$

Therefore we have $T_{2}^{l} \in \mathcal{D}^{\prime}\left(\mathbb{C}^{2} \backslash\{0\}\right)_{\text {even,-2 }}^{H}$ for any $l \in \mathbb{N}$. Furthermore, we have $\operatorname{dim} \mathcal{D}^{\prime}\left(\mathbb{C}^{2} \backslash\{0\}\right)_{\text {even, }-2}^{H}=\infty$ because $\left\{T_{2}^{l}\right\}_{l \in \mathbb{N}}$ are linearly independent. Thus $H$ satisfies 2) by Corollary 2.3. Therefore the proof of the case of $n=2$ completes.

Remark 4.7. For $n=2$, the dimension of $\mathcal{D}^{\prime}\left(\mathbb{C}^{2} \backslash\{0\}\right)_{\text {even },-\lambda}^{H}$ is finite-dimensional for generic $\lambda \in \mathbb{C}$. Indeed one can show that

$$
\operatorname{dim} \mathcal{D}^{\prime}\left(\mathbb{C}^{2} \backslash\{0\}\right)_{\text {even },-\lambda}^{H} \leq 2 \quad \text { for } \lambda \in \mathbb{C} \backslash\{2\} .
$$

Finally, we discuss the supports of elements of $\mathcal{D}^{\prime}\left(G / Q, \chi_{\lambda}\right)^{H}$. If $\lambda \notin 2 \mathbb{N}+4$, we have $\operatorname{supp}\left(T_{\lambda}^{l}\right)=\operatorname{cl}\left(Y_{2 n-3}\right)$ by (4.8). Here $\operatorname{cl}\left(Y_{2 n-3}\right)$ denotes the closure of $Y_{2 n-3}$ in $X$ (See (4.6) for the definition of $Y_{2 j-1} \subset X$ for $j \in\{1,2, \ldots, n\}$ and hereafter we regard as $Y_{2 j-1} \subset G / Q$ by $X \simeq G / Q$ in (4.5)). We put $X_{j}:=\operatorname{cl}\left(Y_{2 j-1}\right) \subset X$. Then we have

$$
\operatorname{dim}\left(\mathcal{D}_{X_{n-1}}^{\prime}\left(G / Q, \chi_{\lambda}\right)^{H} / \mathcal{D}_{X_{n-2}}^{\prime}\left(G / Q, \chi_{\lambda}\right)^{H}\right)=\infty,
$$

where $\mathcal{D}_{X_{j-1}}^{\prime}\left(G / Q, \chi_{\lambda}\right)^{H}:=\left\{F \in \mathcal{D}^{\prime}\left(G / Q, \chi_{\lambda}\right)^{H} \mid \operatorname{supp}(F) \subset X_{j-1}\right\}$. Furthermore, the following statement holds more generally:

Proposition 4.8. Suppose $n \geq 3$. Let $G$ and $Q$ be as in Theorem 1.7. Then for any $j \in\{2,3, \ldots, n-1\}$, we have

$$
\operatorname{dim}\left(\mathcal{D}_{X_{j}}^{\prime}\left(G / Q, \chi_{\lambda}\right)^{H} / \mathcal{D}_{X_{j-1}}^{\prime}\left(G / Q, \chi_{\lambda}\right)^{H}\right)=\infty
$$

for any $\lambda \in \mathbb{C} \backslash(2 \mathbb{N}+2+2 n-2 j)$.

Proof. Let $D_{j}$ be a real analytic vector field on $\mathbb{C}^{n} \simeq \mathbb{R}^{2 n}$ given by $D_{j}:=$ $\bar{z}_{j-1} \frac{\partial}{\partial \bar{z}_{j}}+z_{j} \frac{\partial}{\partial z_{j+1}}$. For $l \in \mathbb{N}$, we define a distribution $T_{\lambda, j}^{l} \in \mathcal{D}^{\prime}\left(\mathbb{C}^{n} \backslash\{0\}\right)$ with holomorphic parameter $\lambda \in \mathbb{C}$ by

$$
T_{\lambda, j}^{l}(z):=\frac{1}{\Gamma\left(n-j+1-\frac{\lambda}{2}\right)} D_{j}^{l}\left(\left|z_{j}\right|^{2(n-j)-\lambda} \prod_{k=j+1}^{n} \delta\left(z_{k}, \bar{z}_{k}\right)\right) .
$$


Then we have $T_{\lambda, j}^{l} \in \mathcal{D}^{\prime}\left(\mathbb{C}^{n} \backslash\{0\}\right)_{\text {even },-\lambda}^{H} \simeq \mathcal{D}^{\prime}\left(G / Q, \chi_{\lambda}\right)^{H}$ in the same way as the case of $T_{\lambda}^{l}$. Moreover $\operatorname{supp}\left(T_{\lambda, j}^{l}\right)=\operatorname{cl}\left(Y_{2 j-1}\right)=X_{j}$ follows easily from (4.13) if $\lambda \in \mathbb{C} \backslash(2 \mathbb{N}+2+2 n-2 j)$. This completes the proof of Proposition 4.8.

Remark 4.9. Let $G_{\mathbb{C}}, Q_{\mathbb{C}}$ and $H_{\mathbb{C}}$ be complexifications of $G, Q$ and $H$, respectively. Then if $\#\left(H_{\mathbb{C}} \backslash G_{\mathbb{C}} / Q_{\mathbb{C}}\right)<\infty$, we have $\operatorname{dim} \mathcal{D}^{\prime}\left(\mathbb{C}^{n} \backslash\{0\}\right)_{\text {even, }-\lambda}^{H}<\infty$ for any $\lambda \in \mathbb{C}$ by the general theory of holonomic systems due to Sato-KashiwaraKawai [6. Theorems 5.1.7, and 5.1.12]. Therefore we have $\#\left(H_{\mathbb{C}} \backslash G_{\mathbb{C}} / Q_{\mathbb{C}}\right)=\infty$ because $\operatorname{dim} \mathcal{D}^{\prime}\left(\mathbb{C}^{n} \backslash\{0\}\right)_{\text {even },-\lambda}^{H}=\infty$ by Proposition 4.6. Alternatively we can show that $\#\left(H_{\mathbb{C}} \backslash G_{\mathbb{C}} / Q_{\mathbb{C}}\right)=\infty$ by direct calculation as below.

Proposition 4.10. Suppose $G, Q$ are as in Theorem 1.7, and $H$ is the subgroup of $G$ defined in (4.4). Let $G_{\mathbb{C}}, Q_{\mathbb{C}}$ and $H_{\mathbb{C}}$ be complexifications of $G, Q$ and $H$, respectively. Then if $n \geq 2$, we have $\#\left(H_{\mathbb{C}} \backslash G_{\mathbb{C}} / Q_{\mathbb{C}}\right)=\infty$.

Before the proof of Proposition 4.10, we discuss the complexifications of $\mathbb{C}$ and $R_{\varepsilon}$ in order to make calculation clear. We write $\overline{\mathbb{C}}$ for the complex conjugate space of $\mathbb{C}$, that is, $\overline{\mathbb{C}}=\mathbb{C}$ as a set, and scalar multiplication of $c \in \mathbb{C}$ given by $c \cdot v:=\bar{c} v$ for $v \in \overline{\mathbb{C}}$. Then the complexification $\mathbb{C} \otimes_{\mathbb{R}} \mathbb{C}$ of $\mathbb{C}$ is isomorphic to $\mathbb{C} \oplus \overline{\mathbb{C}}$ as a $\mathbb{C}$-algebra by the following map:

$$
e_{-} \frac{a \otimes 1}{2}+e_{+} \frac{c \otimes 1}{2} \mapsto(a, c) \quad(a, c \in \mathbb{C}),
$$

where $e_{ \pm}:=1 \otimes 1 \pm i \otimes i \in \mathbb{C} \otimes_{\mathbb{R}} \mathbb{C}$. Here the multiplication of $\mathbb{C} \otimes_{\mathbb{R}} \mathbb{C}$ is given by $(a \otimes b) \cdot(c \otimes d)=a c \otimes b d$. Similarly, we define an isomorphism $R_{\varepsilon} \otimes_{\mathbb{R}} \mathbb{C}=(\mathbb{C} \oplus \mathbb{C} \varepsilon) \otimes_{\mathbb{R}} \mathbb{C} \stackrel{\sim}{\longrightarrow}(\mathbb{C} \oplus \overline{\mathbb{C}}) \oplus(\mathbb{C} \oplus \overline{\mathbb{C}}) \varepsilon$ as a $\mathbb{C}$-algebra by

$$
e_{-}^{\prime} \frac{(a+b \varepsilon) \otimes 1}{2}+e_{+}^{\prime} \frac{(c+d \varepsilon) \otimes 1}{2} \mapsto(a, c)+(b, d) \varepsilon \quad(a, b, c, d \in \mathbb{C}),
$$

where $e_{ \pm}^{\prime}:=1 \otimes 1 \pm i \otimes i \in(\mathbb{C} \oplus \mathbb{C} \varepsilon) \otimes_{\mathbb{R}} \mathbb{C}$. Then the multiplication on $(\mathbb{C} \oplus \overline{\mathbb{C}}) \oplus(\mathbb{C} \oplus \overline{\mathbb{C}}) \varepsilon$ induced from this isomorphism is given below,

$((a, c)+(b, d) \varepsilon)\left(\left(a^{\prime}, c^{\prime}\right)+\left(b^{\prime}, d^{\prime}\right) \varepsilon\right)=\left(a a^{\prime}+b \overline{d^{\prime}}, c c^{\prime}+d \overline{b^{\prime}}\right)+\left(a b^{\prime}+b \overline{c^{\prime}}, c d^{\prime}+d \overline{a^{\prime}}\right) \varepsilon$,

where $(a, c)+(b, d) \varepsilon,\left(a^{\prime}, c^{\prime}\right)+\left(b^{\prime}, d^{\prime}\right) \varepsilon \in(\mathbb{C} \oplus \overline{\mathbb{C}}) \oplus(\mathbb{C} \oplus \overline{\mathbb{C}}) \varepsilon$. Hereafter we identify $R_{\varepsilon} \otimes_{\mathbb{R}} \mathbb{C}$ with $(\mathbb{C} \oplus \overline{\mathbb{C}}) \oplus(\mathbb{C} \oplus \overline{\mathbb{C}}) \varepsilon$ via (4.15). For the proof of Proposition 4.10, we need:

Lemma 4.11. The complexification of the representation of $R_{\varepsilon}$ on $\mathbb{C}$ defined in (4.2) is given below under the identifications of (4.14) and (4.15),

$$
((a, c)+(b, d) \varepsilon) \cdot(z, w)=(a z, c w)+(b \bar{w}, d \bar{z}),
$$

where $(a, c)+(b, d) \varepsilon \in R_{\varepsilon} \otimes_{\mathbb{R}} \mathbb{C}$ and $(z, w) \in \mathbb{C} \oplus \overline{\mathbb{C}} \simeq \mathbb{C} \otimes_{\mathbb{R}} \mathbb{C}$.

This follows from easy calculation, hence we omit the proof. 
Proof of Proposition 4.10, $M_{n}\left(R_{\varepsilon} \otimes \mathbb{C}\right)$ acts on $(\mathbb{C} \oplus \overline{\mathbb{C}})^{n} \simeq \mathbb{C}^{n} \otimes \mathbb{C}$ by left multiplication. This action induces $\iota_{\mathbb{C}}: M_{n}\left(R_{\varepsilon} \otimes \mathbb{C}\right) \sim M_{2 n}(\mathbb{C})$ in the same way as $\iota$ in (4.3). Then the complexification of $H$ in $M_{n}\left(R_{\varepsilon} \otimes \mathbb{C}\right)$ is the following:

$$
H_{\mathbb{C}}:=\left\{h^{a}(\boldsymbol{A}):=\left(\begin{array}{cccc}
\left(e^{i a}, e^{i \bar{a}}\right) & A_{1} \varepsilon & \cdots & A_{n-1} \varepsilon^{n-1} \\
& \left(e^{i a}, e^{i \bar{a}}\right) & \ddots & \vdots \\
& & \ddots & A_{1} \varepsilon \\
& & & \left(e^{i a}, e^{i \bar{a}}\right)
\end{array}\right) \mid \boldsymbol{A} \in(\mathbb{C} \oplus \overline{\mathbb{C}})^{n-1}\right\},
$$

where $\boldsymbol{A}=\left(A_{1}, \ldots, A_{n-1}\right) \in(\mathbb{C} \oplus \overline{\mathbb{C}})^{n-1}$. Similarly to the case of $H$ in (4.4), $\iota\left(H_{\mathbb{C}}\right)$ is a subgroup of $G_{\mathbb{C}}=S L(2 n, \mathbb{C})$ and we may identify $H_{\mathbb{C}}$ in $M_{n}\left(R_{\varepsilon} \otimes \mathbb{C}\right)$ with $\iota_{\mathbb{C}}\left(H_{\mathbb{C}}\right)$ in $G_{\mathbb{C}}=S L(2 n, \mathbb{C})$. Let $\mathbb{C}^{\times}:=G L(1, \mathbb{C})$ act on $(\mathbb{C} \oplus \overline{\mathbb{C}})^{n}$ by scalar multiplication. Then, for $c \in \mathbb{C}^{\times}$and $\left(\left(z_{1}, w_{1}\right), \ldots,\left(z_{n}, w_{n}\right)\right) \in(\mathbb{C} \oplus \overline{\mathbb{C}})^{n}$, we have

$$
c \cdot\left(\left(z_{1}, w_{1}\right), \ldots,\left(z_{n}, w_{n}\right)\right)=\left(\left(c z_{1}, \bar{c} w_{1}\right), \ldots,\left(c z_{n}, \bar{c} w_{n}\right)\right) .
$$

We put $X_{\mathbb{C}}:=\left((\mathbb{C} \oplus \overline{\mathbb{C}})^{n} \backslash\{0\}\right) / \mathbb{C}^{\times}$. By regarding $(\mathbb{C} \oplus \overline{\mathbb{C}})^{n}$ as $\mathbb{C}^{2 n}$, we have $X_{\mathbb{C}} \simeq \mathbb{C P}^{2 n-1} \simeq G_{\mathbb{C}} / Q_{\mathbb{C}}$ and these isomorphisms induce a bijection:

$$
H_{\mathbb{C}} \backslash X_{\mathbb{C}} \simeq H_{\mathbb{C}} \backslash G_{\mathbb{C}} / Q_{\mathbb{C}}
$$

On the other hand, the action of $H_{\mathbb{C}}$ on $(\mathbb{C} \oplus \overline{\mathbb{C}})^{n}$ is given below by Lemma 4.11 (See (4.16) for the definition of $h^{a}(\boldsymbol{A}) \in H_{\mathbb{C}}$ ),

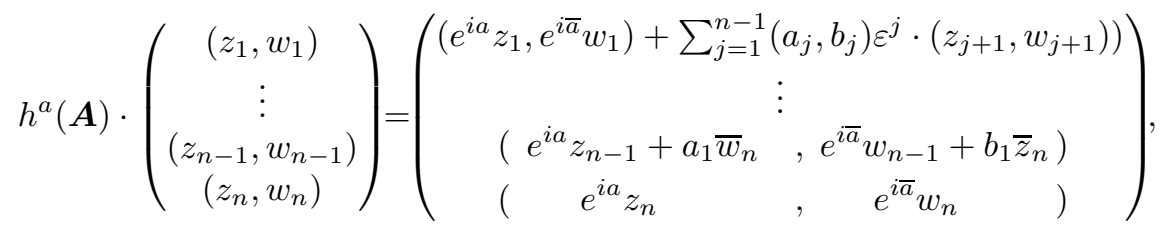

where $a \in \mathbb{C}, \boldsymbol{A}=\left(A_{1}, \ldots, A_{n-1}\right)=\left(\left(a_{1}, b_{1}\right), \ldots,\left(a_{n-1}, b_{n-1}\right)\right) \in(\mathbb{C} \oplus \overline{\mathbb{C}})^{n-1}$ and $\left(\left(z_{1}, w_{1}\right), \ldots,\left(z_{n}, w_{n}\right)\right) \in(\mathbb{C} \oplus \overline{\mathbb{C}})^{n}$. For $\zeta \in \mathbb{C}$, we define a complex $(2 n-3)$ dimensional submanifold of $X_{\mathbb{C}}$ by

$$
Y_{2 n-3}^{\zeta}:=\left\{\left(z_{j}, w_{j}\right)_{j=1}^{n} \in(\mathbb{C} \oplus \overline{\mathbb{C}})^{n} \mid w_{n}=0, z_{n} \neq 0, z_{n-1}=\zeta z_{n}\right\} / \mathbb{C}^{\times} \subset X_{\mathbb{C}}
$$

Then for any $\zeta \in \mathbb{C}$, the group $H_{\mathbb{C}}$ leaves $Y_{2 n-3}^{\zeta}$ invariant, and in fact it acts transitively. Moreover if $\zeta \neq \mu, Y_{2 n-3}^{\zeta}$ and $Y_{2 n-3}^{\mu}$ have no intersection. Therefore we have $\#\left(H_{\mathbb{C}} \backslash G_{\mathbb{C}} / Q_{\mathbb{C}}\right)=\#\left(H_{\mathbb{C}} \backslash X_{\mathbb{C}}\right)=\infty$.

\section{Ackowledgement}

The author is grateful to Professor Toshiyuki Kobayashi for his much helpful advice and constant encouragement and thanks my parents for their support. 


\section{References}

[1] A. Aizenbud, D. Gourevitch, Schwartz functions on Nash manifolds, Int. Math. Res. Not. IMRN 5 (2008), Art. ID rnm 155, 37 pp.

[2] A. Aizenbud, D. Gourevitch, A. Minchenko, Holonomicity of relative characters and applications to multiplicity bounds for spherical pairs, arXiv:1501.01479v1, to appear in Selecta Math.

[3] F. Bien, Orbit, multiplicities, and differential operators, Contemp. Math. 145 (1993), Amer. Math. Soc. 199-227.

[4] I. M. Gelfand, G. E. Shilov, Generalized functions. Vol. I: Properties and operations, Academic Press (1964), New York xvii+423 pp.

[5] Harish-Chandra, Representations of semisimple Lie groups. II, Trans. Amer. Math. Soc. 76 (1954), 26-65.

[6] M. Kashiwara, Systems of Microdifferential Equations, Progr. Math. 34 (1983), Birkhäuser, xv+159 pp.

[7] B. Kimelfeld, Homogeneous domains in flag manifolds, J. Math. Anal. Appl. 121 (1987), 506-588.

[8] T.Kobayashi, Introduction to harmonic analysis on real spherical homogeneous spaces, Proceedings of the 3rd Summer School on Number Theory Homogeneous Spaces and Automorphic Forms in Nagano (F. Sato, ed.), 1995, 22-41 (in Japanese).

[9] T. Kobayashi, Shintani functions, real spherical manifolds, and symmetry breaking operators, Developments in Mathematics 37 (2014), 127-159.

[10] T. Kobayashi, T. Oshima, Finite multiplicity theorems for induction and restriction, Adv. Math. 248 (2013), 921-944.

[11] T. Kobayashi, B. Speh, Symmetry Breaking for Representations of Rank One Orthogonal Groups, Mem. Amer. Math. Soc. 238 (2015), 118 pp.

[12] T. Kobayashi, T. Yoshino, Compact Clifford-Klein forms of symmetric spaces-revisited, Pure and Appl. Math. Quarterly 1 (2005), 603-684.

[13] T. Matsuki, Orbits on flag manifolds, Proceedings of the International Congress of Mathematicians, Kyoto 1990, Vol. II (1991), Springer-Verlag, 807-813. 